\title{
THE CHALLENGES OF THE SUPER-MICRO MO DISK (Invited)
}

\author{
Y. TSUNODA \\ Central Research Laboratory, Hitachi Ltd., 1-280 Higashi-koigakubo Kokubunji, Tokyo 185, Japan
}

\section{Introduction}

Advancement of computers and communication networks are driving the rapid evolution of our information society towards the 21 st Century. As these systems become more sophisticated, an avalanche of image data, document data, and computer data stream can be seen into our midst. In order to properly store, preserve and retrieve all this information, we have to rely on a variety of information storage systems and devices. However, without the access to the very best systems we cannot hope to nurture the growth of our information society in the 21 st Century.

Magneto optical memory(MOM) is one of the promising candidates for replying this demand. Since the first introduction of the product into the market in 1988, considerable progress has been made in both technologies and applications. Evolution of the technology will open completely new professional and personal storage markets in future, featuring such intrinsic advantages of the optical memory as media removability, high storage density, and easiness of mass replication. Aiming at the future ultra large capacity and high throughput products, challenges of the ultra high density magneto optical memory have been pursued in several places.

In this presentation, first of all, I would like to explain the wide range application areas for present and future magneto optical memories. Secondly I will discuss the future storage density increase of the magneto optical memory referring the major possible approaches. Finally I will describe the basic concept of the super-micro MO disk memory which is expected to dominate the future personal storage.

\section{Major application areas of MOM}

Figure 1 shows the major application areas of MOM at present and in the future. A large variety of applications are roughly classified into three major areas. A large scale center file has huge amount of data and rather wide range of data access rate. A middle scale distributed file has the capacity of several GB up to several hundreds GB. A small scale personal file has the capacity of several tens MB up to several GB and requires slower data access rate than the distributed file. These amount of data and data access rate will be increased to one order higher livel in the year 2000. Without drastic improvements of MOM, future information society will be thrown into disorder because of a flood of information.

\section{Storage density increase of MOM}

Figure 2 shows the past and the future trend of the storage density increase of the optical memory, comparing with that of magnetic memory. The storage density of the magnetic memory has been increased to one order higher level during these ten years. This will likely happen in the future, so that 10 times and 100 times higher storage densities than now can be expected in the year 2000 and 2010, respectively. The same ratio of the storage density increase can be expected even for MOM. Eventurally 10 times higher storage density increase can be expected in the year 2000 . The challenges of super high density $10 \mathrm{~Gb} / \mathrm{in}^{2}$ storage have been pursued. Developments of shorter wavelength light sources, high speed magnetic field modulation recording, and high density pit-edge are some examples.

\section{Proposed super-micro MO disk}

Storage density of $10 \mathrm{~Gb} / \mathrm{in}^{2}$ can provide drastic changes on the MOM concept. Figure 3 shows the basic concept of the super-micro MO disk with $10 \mathrm{~Gb} / \mathrm{in}^{2}$ storage density. Total storage 
capacity of the disk with $30 \mathrm{~mm}$ diameter is $1 \mathrm{~GB} .80$ to 90 percent of the capacity is used for storing the prerecorded ROM in which personal distribution data such as journal documents, newspapers, technical drawings, and a variety of public data are included. 10 to 20 percent of the capacity consists of the magneto optical recording area and can be used for editing, creating, and storing the user's own data. This tiny super-micro MO disk can be installed in the drive which is contained in the palmtop computer.

\section{Conclusion}

Evolution of MOM towards the 21st Century has been described. Prospect for the storage density of $10 \mathrm{~Gb} / \mathrm{in}^{2}$ has been examined and the basic concept of the super-micro MO disk has been shown.

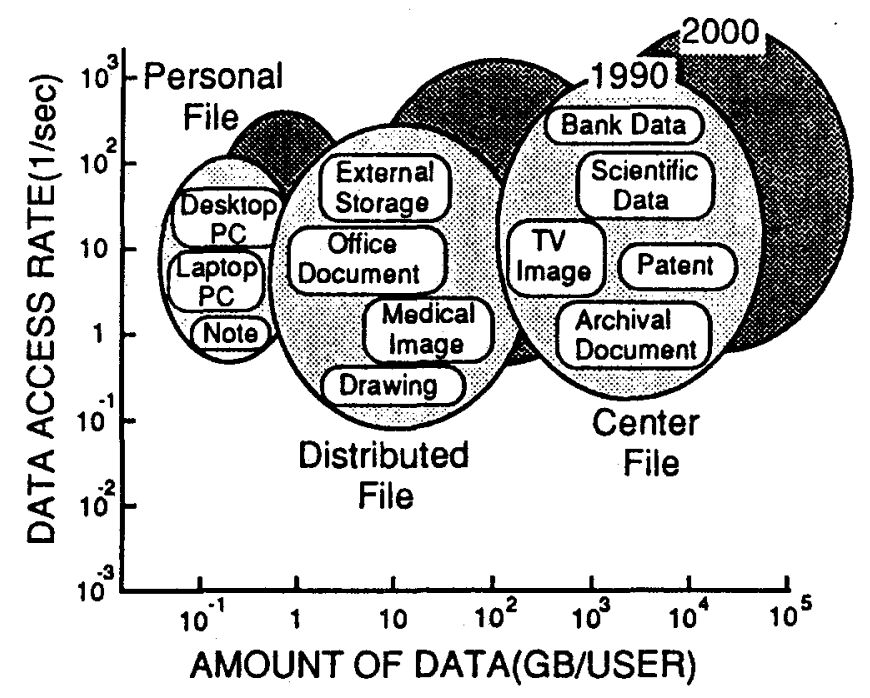

Figure 1. Application areas of MOM

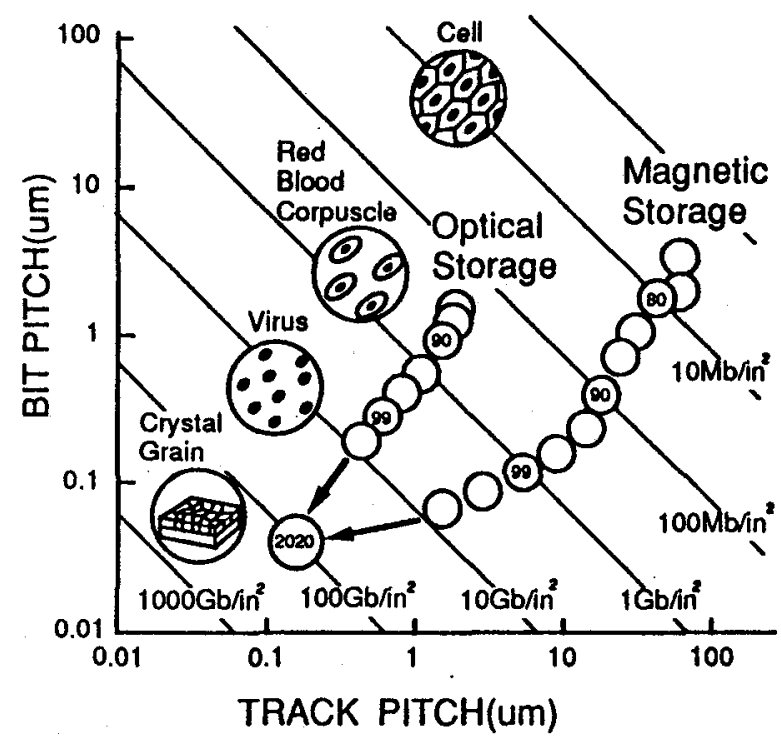

Figure 2. Past and future trends of storage density increase

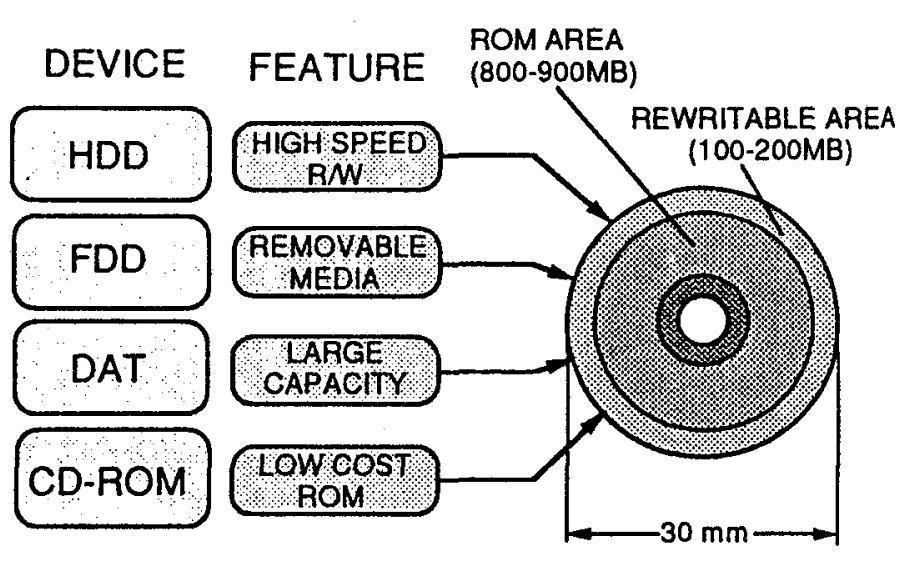

Figure 3. Basic concept of super-micro MO disk 